\title{
The Noise Stability Estimating Method of Perspective Navigation Receiver GNSS Un-manned Aerial Vehicle
}

\author{
Alexey V. Korovin, \\ Vladimir A. Mironov, Alexandr A. Novikov, \\ Alexey Yu. Truscinskiy and Yuriy L. Fateev* \\ Military Education and Research Centre of Military-Air Forces \\ "Military-Air Academy \\ Named After Professor N.E. Zhukovsky and Yu.A. Gagarin» \\ 54a Starykh Bolshevikov Str., Voronezh, 394064, Russia
}

On the basis of previously synthesized by the authors primary processing channels GPS re-ceiver algorithms, interference and navigation signals models, developed methodology and con-ducted noise studies of channel delay and frequency multi-system navigation receiver global navi-gation satellite systems, performs simultaneous reception and initial processing of different types of navigation signals. As indicators of noise immunity the probability and mean time before tracking the navigation signal.

Keywords: global navigation satellite system, navigation equipment of the consumer, the probability of tracking the navigation signal, the average time before tracking the navigation signal.

Citation: Korovin A.V., Mironov V.A., Novikov A.A., Truscinskiy A.Y., Fateev Yu.L. The noise stability estimating method of perspective navigation receiver GNSS unmanned aerial vehicle, J. Sib. Fed. Univ. Eng. technol., 2016, 9(8), $1162-1171$. DOI: 10.17516/1999-494X-2016-9-8-1162-1171.

(C) Siberian Federal University. All rights reserved

* Corresponding author E-mail address: mirvam@live.ru 


\title{
Методика оценки помехоустойчивости
}

\section{перспективного навигационного приемника ГНСС беспилотного летательного аппарата}

\author{
А.В. Коровин, В.А. Миронов, \\ А.А. Новиков, А.Ю. Трущинский, Ю.Л. Фатеев \\ Военный учебно-научный цеентр Военно-воздушных сил \\ «Военно-воздушная академия \\ имени профессора Н.Е. Жуковского и Ю.А. Гагарина» \\ Россия, 394064, Воронеж, ул. Старых Большевиков, 54 а
}

На основе синтезированных ранее авторами алгоритмов работы каналов первичной обработки навигачионного приемника, моделей помех и навигационных сигналов разработана методика и проведены исследования помехоустойчивости каналов задержки и частоть мультисистемного многоканального навигационного приемника глобальных навигационных спутниковых систем, выполняющего одновременный прием и первичную обработку разных типов навигационных сигналов. В качестве показателей помехоустойчивости определены вероятность и среднее время до срыва слежения за навигационным сигналом.

Ключевые слова: глобальная навигационная спутниковая система, навигачионная аппаратура потребителя, вероятность срыва слежения за навигационным сигналом, среднее время до срыва слежения за навигационным сигналом.

Предположительный состав бортового комплекса навигации (БКН) беспилотного летательного аппарата (БЛА) средней и большой дальности, позволяющего с высокой эффективностью выполнять весь спектр боевых задач, будет включать следующие системы:

- инерциальная навигационная система;

- мультисистемная навигационная аппаратура потребителей (НАП) глобальных навигационных спутниковых систем (ГНСС) [1,2];

- автоматическая радарно-лазерная система отображения обстановки;

- интерференционная система взлета и посадки.

Однако необходимо признать то, что НАП ГНСС как одна из основных систем навигации БКН имеет невысокую помехоустойчивость и подвержена влиянию различного рода непреднамеренных и преднамеренных радиопомех, затенений и переотражений принимаемых сигналов от навигационных спутников (НC). Это в значительной степени снижает боевую эффективность БКН БЛА и, соответственно, его приспособленность к выполнению задач в конкретных условиях боевого применения с учетом огневого, радиоэлектронного и других видов противодействия противника $[3,4]$.

Таким образом, в объеме решения задач оценки эффективности БКН БЛА предлагаются разработанные методики определения статистических характеристик помехоустойчивости НАП ГНСС.

Следовательно, для обоснования необходимых тактико-технических требований образцов НАП ГНСС военного назначения необходимо рассчитать статистические характеристики помехоустойчивости: 
1. Вероятность срыва слежения за навигационным сигналом.

2. Среднее время до срыва слежения за навигационным сигналом.

Входные параметры предлагаемой методики:

- математические модели навигационных сигналов (фазоманипулированный двоичной псевдослучайной последовательностью сигнал (BPSK - binary phase shift keying) и перспективный фазоманипулированный двоичной псевдослучайной последовательностью сигнал с дополнительной меандровой модуляцией (BOC - binary offset carrier);

- математическая модель помехи (частотно-модулированная шумом (ЧМШ) помеха (белый гауссовский шум (БГШ).

Основные допущения и ограничения в данной методике:

- поляризация навигационных сигналов производится ортогональными тригонометрическими функциями;

- навигационные сигналы излучаются на одной несущей частоте;

- рассогласование опорного и принятого сигналов принималось равным нулю;

- мощность сигнала на входе цифровой модели приемника задавалась на уровне $-160 \mathrm{~dB}$;

- не учитывались потери на распространение радиоволн в пространстве;

- ширина спектра помехового сигнала на несущей частоте навигационных сигналов задавалась 5, 10, 15 МГц, на поднесущей частоте навигационных сигналов задавалась соответственно 5 и 2,5 МГц.

Схемотехнические и программные решения при построении современных навигационных приемников позволяют обрабатывать все доступные навигационные сигналы - принцип AllinView, благодаря увеличению количества каналов приема и применению цифровой обработки сигнала непосредственно на несущей частоте. В связи с этим нет необходимости в изменении конструкции НАП ГНСС, достаточно лишь переустановить программное обеспечение. Но следует отметить, что существующий методический аппарат построения НАП ГНСС ориентирован на алгоритмы первичной обработки отдельных навигационных сигналов и не учитывает возможность одновременной совместной обработки разных типов существующих и перспективных навигационных сигналов ГНСС [1, 5-7].

\section{Основные математические соотношения методики}

В связи с тем что при излучении навигационных сигналов BPSK и ВОС используется модуляция ортогональными тригонометрическими функциями несущей частоты, появляется возможность проводить их совместную первичную обработку. На рис. 1 приведены спектральные плотности мощности навигационных сигналов BPSK, BOC и помехи.

Таким образом, на вход цифровых моделей $[1,5-7]$ мультисистемного многоканального приемника НАП подавалась аддитивная смесь сигнала и помехи вида

$$
\hat{y}(t)=y_{B P S}(t)+y_{B O C}(t)+n(t),
$$

где $n(t)$ - ЧМШ (БГШ). 


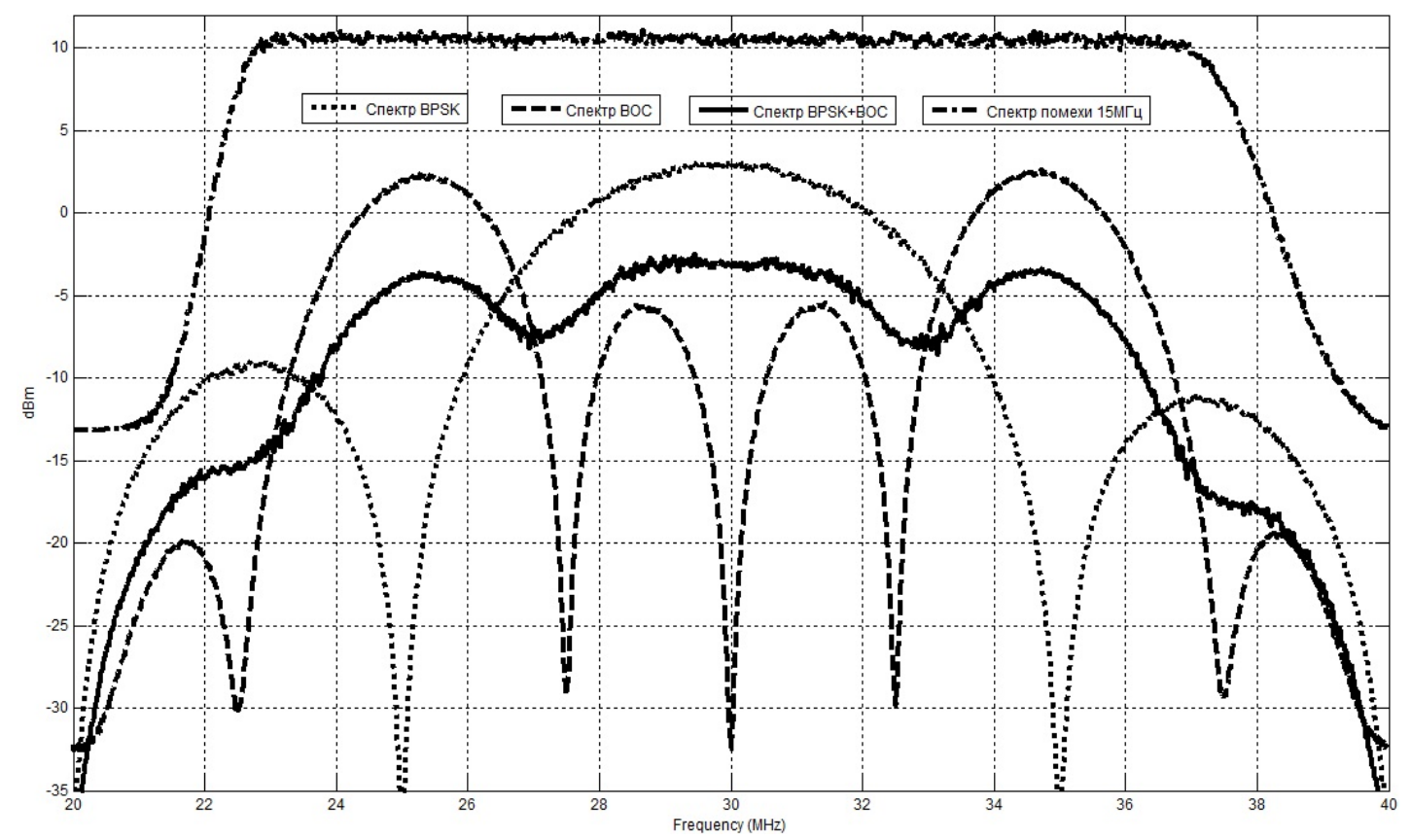

Рис. 1. Спектральные плотности мощности навигационных сигналов и помехи

Напряжения на выходе дискриминаторов следящих систем при воздействии помехи согласно синтезированным алгоритмам работы временного и частотного дискриминаторов [1, 5-7] определяются следующими выражениями:

$\hat{u}_{\text {Дt } \mathrm{BPSK}(\mathrm{BOC})}\left(t_{k}\right)=\frac{\sqrt{\hat{I}_{E B P S K(B O C)}^{2}+\hat{Q}_{E B P S K(B O C)}^{2}}-\sqrt{\hat{I}_{L B P S K(B O C)}^{2}+\hat{Q}_{L B P S K(B O C)}^{2}}}{\sqrt{\hat{I}_{E B P S K(B O C)}^{2}+\hat{Q}_{E B P S K(B O C)}^{2}}+\sqrt{\hat{I}_{L B P S K}^{2}+\hat{Q}_{L B P S K(B O C)}^{2}}}$,

$\hat{u}_{\text {Дi } \tau_{B P S K+B O C}}\left(t_{k}\right)=\frac{\sqrt{\hat{I}_{E B P S K}^{2}+\hat{Q}_{E B P S K}^{2}+\hat{I}_{E B O C}^{2}+\hat{Q}_{E B O C}^{2}}-\sqrt{\hat{I}_{L B P S K}^{2}+\hat{Q}_{L B P S K}^{2}+\hat{I}_{L B O C}^{2}+\hat{Q}_{L B O C}^{2}}}{\sqrt{\hat{I}_{E B B S K}^{2}+\hat{Q}_{E B P S K}^{2}+\hat{I}_{E B O C}^{2}+\hat{Q}_{E B O C}^{2}}+\sqrt{\hat{I}_{L B P S K}^{2}+\hat{Q}_{L B P S K}^{2}+\hat{I}_{L B O C}^{2}+\hat{Q}_{L B O C}^{2}}}$,

$\hat{u}_{\text {Дक } \omega_{B P S K}(B O C)}\left(t_{k}\right)=\hat{I}_{P B P S K(B O C)}(k) Q_{P B P S K(B O C)}(k-1)-\hat{I}_{P B P S K(B O C)}(k-1) Q_{P B P S K(B O C)}(k)$,

$$
\begin{array}{r}
\hat{u}_{\text {Д的 }} \text { BPSK+BOC } \\
\left(t_{k}\right)=\hat{I}_{P B P S K_{P B P S K}}(k) Q_{P B P S K}(k-1)-\hat{I}_{P B P S K}(k-1) Q_{P B P S K}(k)+ \\
+\hat{I}_{P B O C}(k) \hat{Q}_{P B O C}(k-1)-\hat{I}_{P B O C}(k-1) \hat{Q}_{P B O C}(k),
\end{array}
$$

где $\hat{I}$ и $\hat{Q}$ - синфазные и квадратурные, несдвигаемые, опережающие и запаздывающие составляющие при наличии помехи.

Подавая аддитивную смесь сигнала и помехи на вход модели приемника с псевдодоплеровским смещением частоты и рассогласованием сигнала и опорной последовательности, равной нулю, определяем флуктуационные характеристики каналов слежения за задержкой и частотой [8], которые описываются следующими соотношениями: 


$$
\begin{aligned}
& D_{\tau}=\left.M\left[\left(u_{\not \tau}-u(\tau, \hat{\tau})\right)^{2}\right]\right|_{\varepsilon_{\tau}}=0, \\
& D_{\omega}=\left.M\left[\left(u_{\not \omega}-u(\omega, \hat{\omega})\right)^{2}\right]\right|_{\varepsilon_{\omega}}=0 .
\end{aligned}
$$

На рис. 2 и 3 представлены флуктуационные характеристики следящих систем за задержкой и частотой для ширины спектра помехи 5 МГц (a), 10 МГц (б), 15 МГц (в) соответственно.

Вычисленные дисперсии шума на выходе временных и частотных дискриминаторов согласно следующим выражениям:

$$
\begin{aligned}
& D_{\amalg \tau}=\left\{\hat{u}_{\text {Д } \tau}^{2}-\left\{\hat{u}_{\text {Д } \tau}\right\}^{2}\right\}, \\
& D_{\amalg \omega}=\left\{\hat{u}_{\text {Д } \omega}^{2}-\left\{\hat{u}_{\text {Д } \omega}\right\}^{2}\right\}
\end{aligned}
$$

сравниваются с пороговой (в случае нормирования с единицей), и принимается решение о срыве слежения за навигационным сигналом $[1,5,6]$.

Вероятность срыва слежения за навигационным сигналом определяется методом статистических испытаний [9]:

$$
P=\frac{\sum_{i=1}^{N}\left(D_{W i} \geq 1\right)}{N},
$$

где $N$ - количество испытаний.

Регистрирующее устройство моделей исследования оценивает вероятность срыва слежения для различных уровней сигнал-шум, зависимости которых изображены на рис. 4 и 5 соответственно для следящих систем за задержкой и частотой.

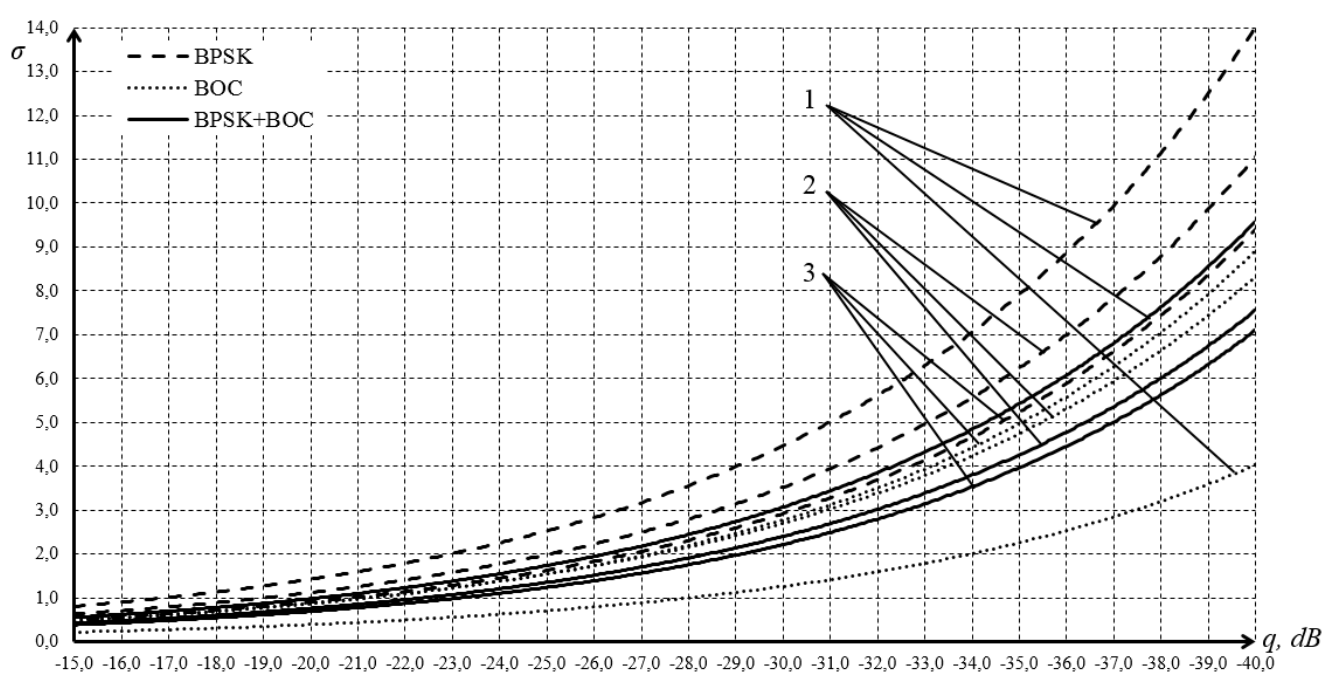

Рис. 2. Флуктуационные характеристики дискриминаторов задержки BPSK, BOC навигационных сигналов для ширины спектра помехи: 1 - 5 МГц; 2 - 10 Мгц; 3 - 15 МГц 


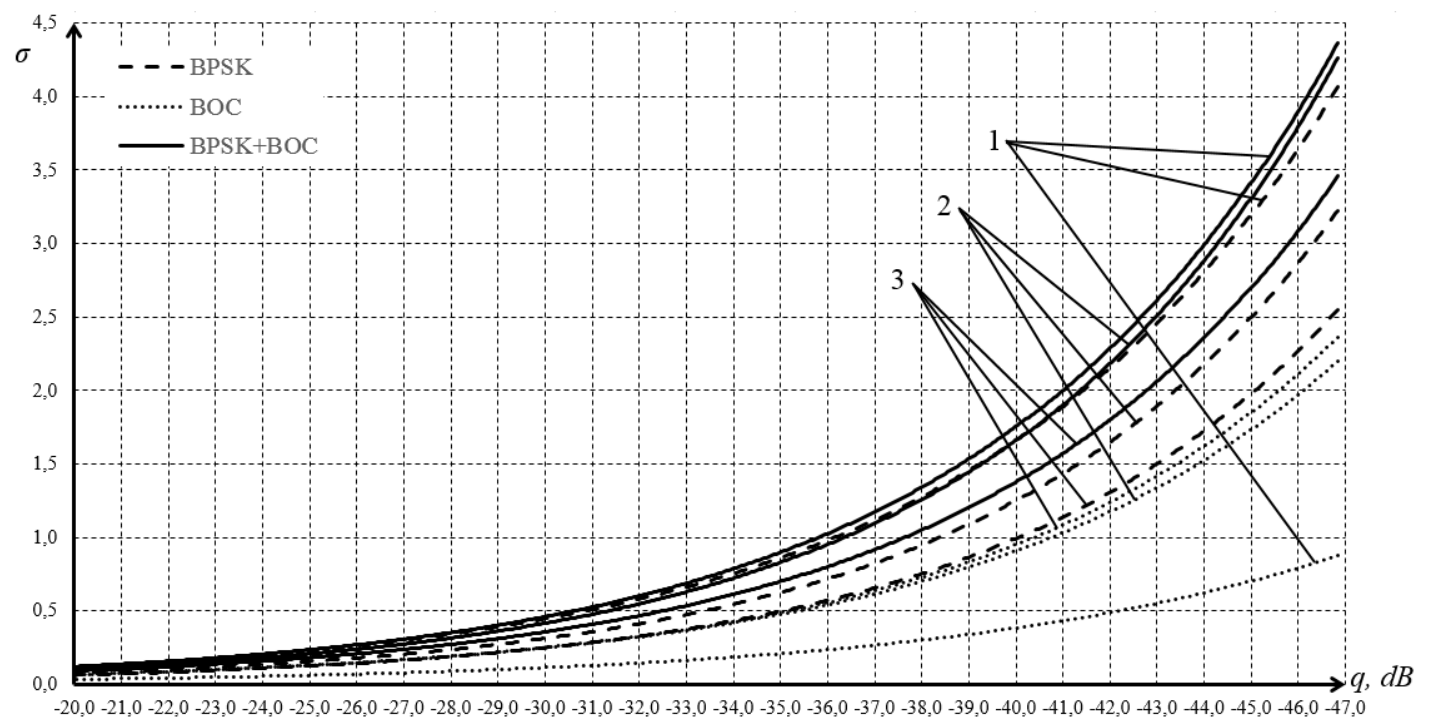

Рис. 3. Флуктуационные характеристики частотных дискриминаторов BPSK, BOC навигационных сигналов для ширины спектра помехи: 1 - 5 МГц; 2 - 10 Мгц; 3 - 15 МГц

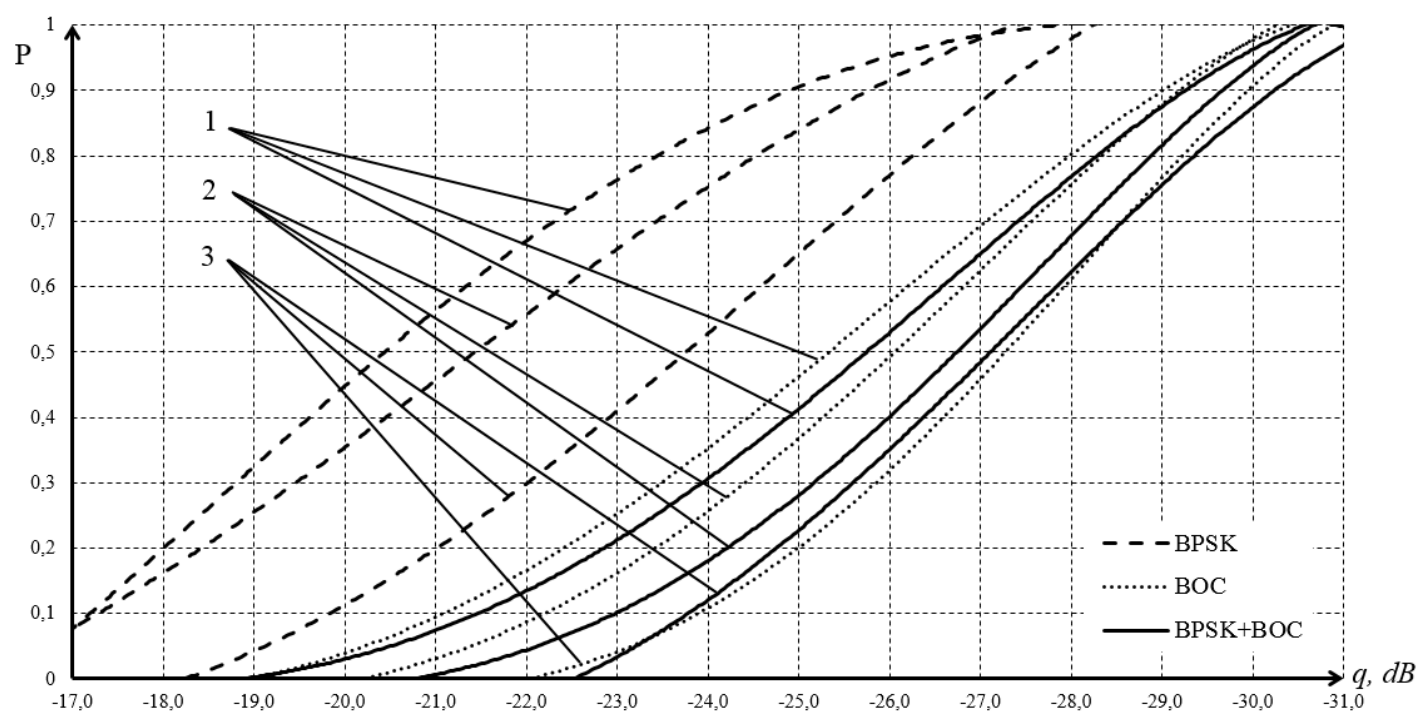

Рис. 4. Зависимости вероятности срыва слежения за BPSK, ВОС навигационными сигналами от отношения сигнал-шум на входе канала слежения за задержкой огибающей для ширины спектра помехи: 1 - 5 МГц; 2 - 10 Мгц; 3 - 15 МГц

Среднее время до срыва слежения за навигационным сигналом определяется как модельное время, в момент которого напряжение на выходе следящей системы при воздействии помехи превышало заданный уровень [10].

На выходе временного и частотного дискриминаторов модели навигационного приемника определяются отсчеты напряжения при воздействии шума и динамики движения объекта из расчета изменения задержки и частоты Доплера навигационного сигнала. Отсчеты напряже- 


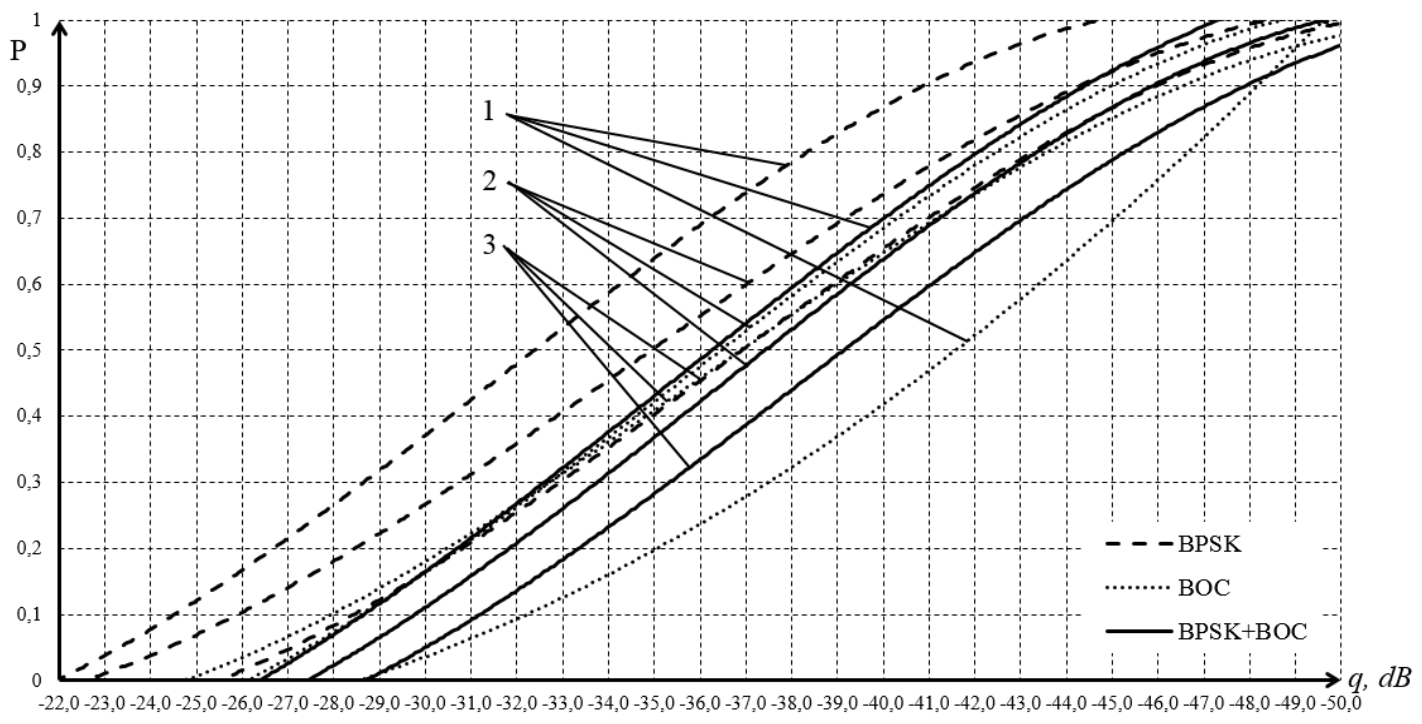

Рис. 5. Зависимости вероятности срыва слежения за BPSK, ВОС навигационными сигналами от отношения сигнал-шум на входе канала слежения за частотой для ширины спектра помехи: $1-5$ МГц; 2 - 10 Мгц; 3 - 15 МГц

ния на выходе следящей системы за задержкой и частотой сравниваются с порогом, который определяется из расчета максимального напряжения рассогласования на выходах временного и частотного дискриминаторов в пределах ширины апертуры раскрыва дискриминационной характеристики. В процессе расчетов модельное время жестко привязывается к реальному времени.

Выражение, описывающее среднее время до срыва слежения за навигационным сигналом, запишем в виде

$$
t_{c p}=\frac{\sum_{i=1}^{N} t_{k}}{N},
$$

где $t_{k}$ - модельное время, в момент которого напряжение на выходе следящей системы при воздействии помехи превышает заданный порог в каждом из $\mathrm{N}$ опытов [9].

Таким образом, при превышении ошибки слежения заданного порога (к примеру, в нашем случае 30 м = ширине взлетно-посадочной полосы) принимается решение о модельном времени $t_{k}$, при котором происходит срыв слежения за навигационным сигналом, жестко привязанному к реальному времени [10].

На рис. 6 и 7 приведены зависимости среднего времени до срыва слежения за навигационным сигналом от отношения сигнал-шум на входе объединённой следящей системы за задержкой и частотой для ширины спектра помехи 5 МГц (a), 10 МГц (б), 15 МГц (8) на центральной частоте и для ширины спектра помехи 2,5 МГц (2) и 5 МГц (д) при воздействии на перспективный навигационный сигнал на поднесущей частоте.

Предложенные методики определения статистических характеристик помехоустойчивости по показателям вероятности срыва слежения и среднего времени до срыва слежения за на- 


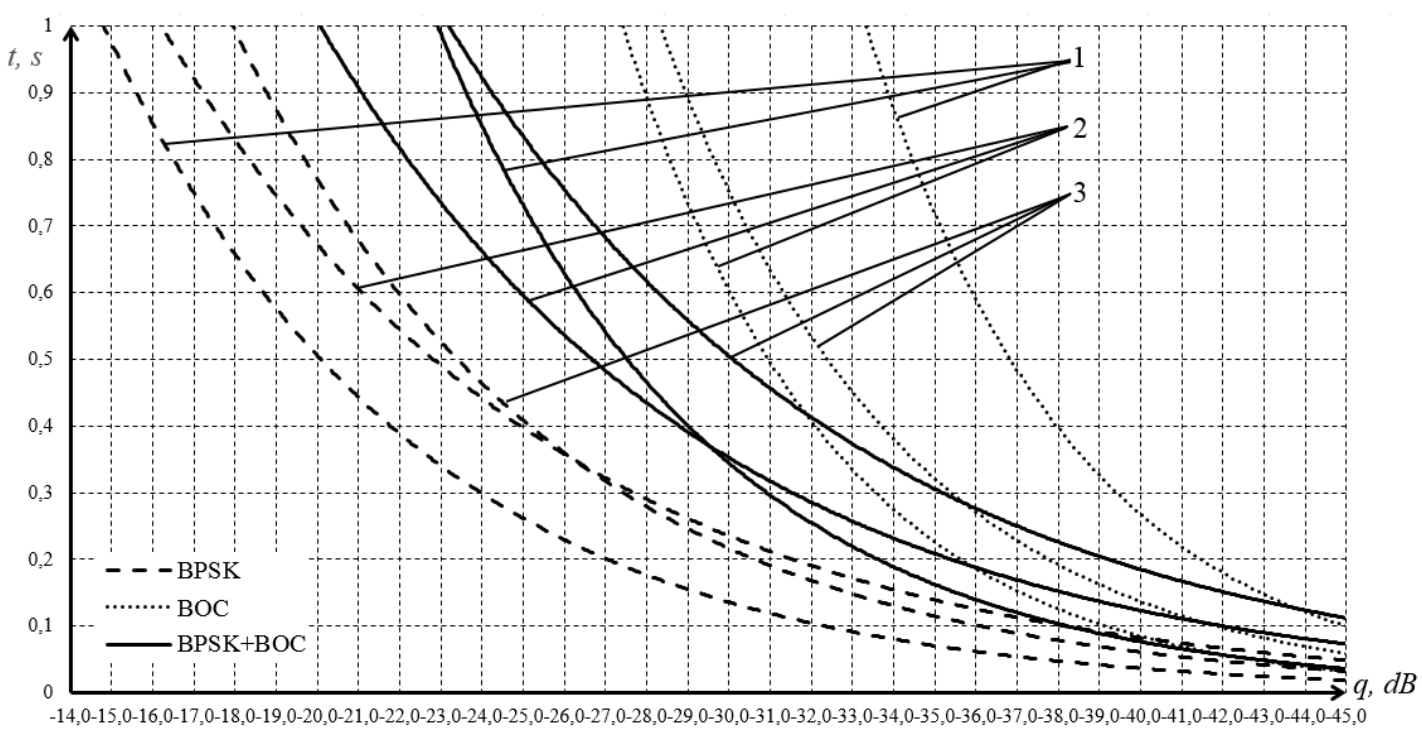

Рис. 6. Зависимости среднего времени срыва слежения за BPSK, BOC навигационными сигналами на несущей частоте от отношения сигнал-шум на входе объединенного канала слежения для ширины спектра помехи: 1 - 5 МГц; 2 - 10 Мгц; 3 - 15 МГц

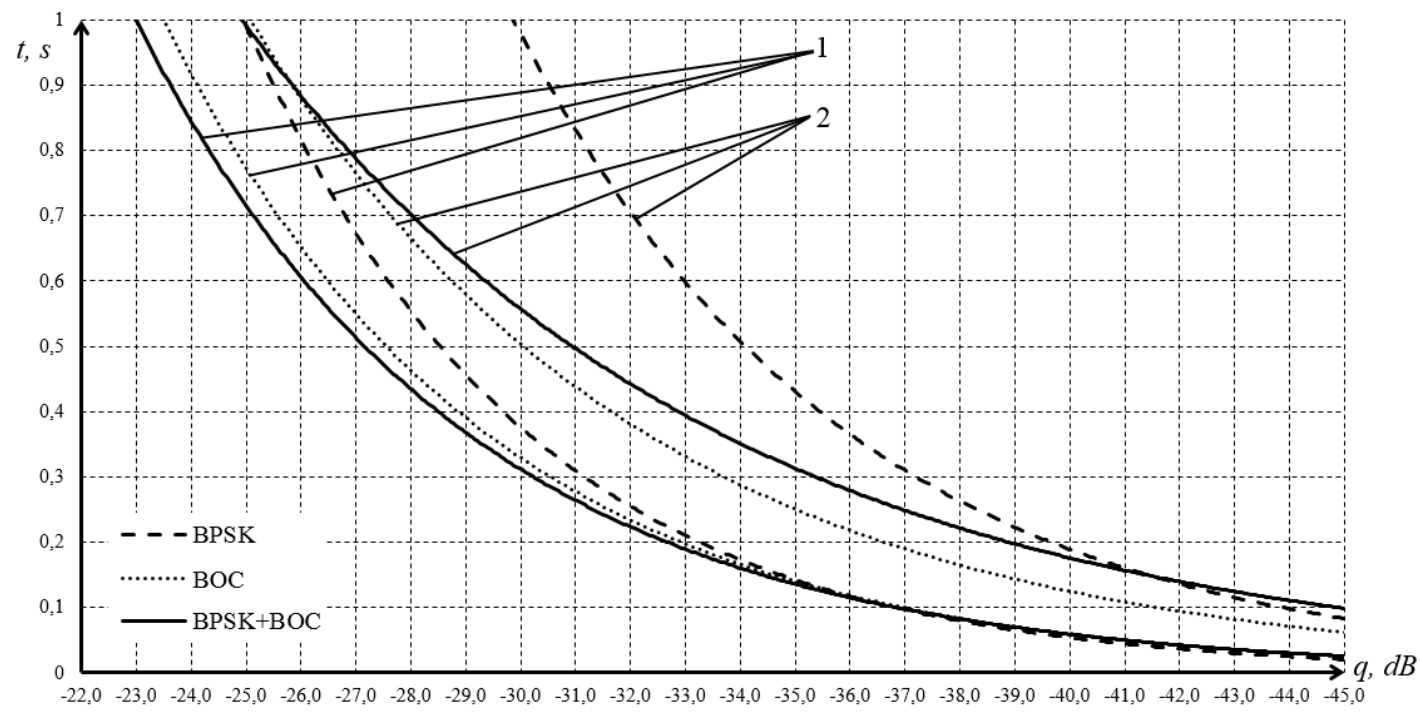

Рис. 7. Зависимости среднего времени срыва слежения за BPSK, BOC навигационными сигналами на поднесущей частоте от отношения сигнал-шум на входе объединенного канала слежения для ширины спектра помехи: 1 - 5 МГц; 2 - 2,5 Мгц

вигационным сигналом перспективной мультисистемной многоканальной НАП ГНСС и анализ приведенных выше зависимостей при воздействии ЧМШ помехи различной интенсивности и различной шириной спектра, соизмеримой с шириной спектра исследуемых навигационных сигналов, позволяют утверждать об увеличении помехоустойчивости перспективного навигационного приемника ГНСС на $3 \div 6$ дБ $[1,2,5,6,10]$, а соответственно, и боевой эффективности БНК БЛА при радиоэлектронном противодействии. 


\section{Список литературы}

[1] Миронов В.А., Коровин А.В., Неровный В.В., Новиков А.А. Разработка и исследование математической модели каналов одновременного слежения за задержкой и частотой навигационного приемника ГНСС при совместной обработке разных типов навигационных сигналов в условиях помех. Сб. тр. XIV Междунар. науч.-техн. конф. «Радиолокация, навигащия, связь». Воронеж: ВГУ, 2014, 1035-1045 [Mironov V.A., Korovin A.V., Nerovnyi V.V., Novikov A.A. Development and research of mathematical model of simultaneous channels of tracking delay and frequency GNSS navigation receiver in joint processing of different types of navigation signals in conditions of interference. Radiolocation, navigation, communication. Voronezh, VGU, 2014, 10351045 (in Russian)]

[2] Авдеев М.В., Журавлев А.В., Коровин А.В., Неровный В.В. Повышение помехозащищенности навигационной аппаратуры потребителей спутниковых радионавигационных систем за счет совместной обработки различного типа навигационных сигналов. Сб. тр. XIV Междунар. науч.-техн. конф. «Радиолокащия, навигащия, связь». Воронеж: ВГУ, 2010, 1583-1589 [Avdeev M.V., Zhuravlev A.V., Korovin A.V., Nerovnyi V.V. Improving the noise immunity of navigation equipment of consumers of satellite navigation systems due to the joint processing of different types of navigation signals. Radiolocation, navigation, communication. Voronezh, VGU, 2010, 1583-1589 (in Russian)]

[3] Ярлыков М.С., Богачев А.С., Меркулов В.И., Дрогалин В.В. Радиоэлектронные комплексы навигации, прищеливания и управления вооружением летательных аппаратов. Т. 1. Теоретические основы / под ред. М.С. Ярлыкова. М.: Радиотехника, 2012. 504 с. [Iarlykov M.S., Bogachev A.S., Merkulov V.I., Drogalin V.V. Radio-electronic systems of navigation, sighting and weapons control aircraft. V. 1. Theoretical foundations. Ed. by M.S. Iarlykov. Moscow, Radiotekhnika, 2012. 504 p. (in Russian)]

[4] Васильев В.Н. Надежность комплексов авиационного радиоэлектронного оборудования. Надежность и техническая диагностика комплексов авиационного радиоэлектронного оборудования: В 3 ч. М.: ВВИА, 2003. Ч. 1. 95 с. [Vasilev V.N. The reliability of the complexes avionics. Reliability and technical diagnostics of aviation complexes of radio-electronic equipment. In 3 parts. Moscow, VVIA, 2003. Part. 1. 95 p. (in Russian)]

[5] Коровин А.В., Миронов В.А., Неровный В.В., Новиков А.А. Разработка и исследование помехоустойчивости модели канала совместного слежения за задержкой различного типа сигналов в навигационной аппаратуре потребителей глобальных навигационных спутниковых систем. Электромагнитные волны и электронные системы, 2015, 2, 4-10 [Korovin A.V., Mironov V.A., Nerovnyi V.V., Novikov A.A. Development and research of noise immunity of the model channel joint delay tracking of different type of signals in the navigation equipment of global navigation satellite systems. Electromagnetic waves and electronic systems, 2015, 2, 4-10 (in Russian)]

[6] Коровин А.В., Миронов В.А., Неровный В.В., Новиков А.А. Разработка и исследование частотных характеристик совместной обработки разных типов навигационных сигналов. Электромагнитные волны и электронные системы, 2015, 3, 11-16 [Korovin A.V., Mironov V.A., Nerovnyi V.V., Novikov A.A. Development and research of frequency characteristics of the coprocessing of different types of navigation signals. Electromagnetic waves and electronic systems, 2015, 3, 11-16 (in Russian)] 
[7] Коровин А.В., Миронов В.А., Новиков А.А., Рябков П.В., Трущинский А.Ю. Частотные дискриминаторы перспективных навигационных приемников ГНСС ГЛОНАСС для малых и больших отношений сигнал/шум. Теория и техника радиосвязи, 2015, 4, 71-78 [Korovin A.V., Mironov V.A., Novikov A.A., Riabkov P.V., Trushchinskii A.Iu. Frequency discriminators promising navigation receivers GLONASS for large and small relations signal/noise. Theory and technique of radio communication, 2015, 4, 71-78 (in Russian)]

[8] ГЛОНАСС. Принципы построения и функиионирования / под ред. А.И. Перова, В.Н. Харисова. Изд. 4-е, перераб. и доп. М.: Радиотехника, 2010. 800 с. [GLONASS. The principles of construction and operation. Ed. by A.I. Perov, V.N. Kharisov. Issue 4. Moscow, Radiotekhnika, 2010. 800 p. (in Russian)]

[9] Тихонов В.И., Ефименко В.С., Журавлев А.Г. Статистическая теория радиоэлектронных систем / под ред. В.И. Тихонова. М.: ВАТУ, 2002. 758 с. [Tikhonov V.I., Efimenko V.S., Zhuravlev A.V. Statistical theory of electronic systems. Ed. by V.I. Tikhonov. Moscow, VATU, 2002. 758 p. (in Russian)]

[10] Авдеев М.В., Журавлев А.В., Коровин А.В., Новиков А.А., Рябков П.В. Исследование помехоустойчивости перспективной НАП ГНСС ГЛОНАСС по показателю среднего времени до срыва слежения за частотой навигационного сигнала. Теория и техника радиосвязи, 2015, 4, 11-17 [Avdeev M.V., Zhuravlev A.V., Korovin A.V., Novikov A.A., Riabkov P.V. Research of noise stability of perspective NAP GLONASS in terms of average time to failure of the tracking frequency of the navigation signal. Theory and technique of radio communication, 2015, 4, 11-17 (in Russian)] 